\title{
TÉCNICA MODIFICADA PARA LA RESECCIÓN TRANSURETRAL DE PRÓSTATA CON DRENAJE SUPRAPÚBICO Y ANESTESIA LOCAL
}

\author{
J.M. SÁNCHEZ ZALABARDO, M.A. SÁNCHEZ ELIPE, O. REGOJO ZAPATA, \\ A. ELIZALDE BENITO, J. VALLE GERHOLD, J.A. LÓPEZ LÓPEZ, J.G. VALDIVIA URÍA
}

Servicio de Urología. Hospital Clínico Universitario Lozano Blesa. Zaragoza.

Actas Urol Esp. 27 (3): 216-220, 2003

\section{RESUMEN}

"TÉCNICA MODIFICADA PARA LA RESECCIÓN TRANSURETRAL DE PRÓSTATA CON DRENAJE SUPRAPÚBICO Y ANESTESIA LOCAL"

INTRODUCCIÓN: Describimos una técnica para la resección transuretral (RTU) de próstata combinando el uso de anestesia local con un tubo de Amplatz suprapúbico en pacientes con alto riesgo quirúrgico.

MATERIAL Y MÉTODOS: Nuestro estudio comprende 32 pacientes sometidos a RTU de próstata con un tubo de Amplatz suprapúbico de $30 \mathrm{Fr}$ bajo anestesia local. Esta técnica se indicó en aquellos casos con alto riesgo quirúrgico. La edad media fue de 70 años (61-82 años). Evaluamos el riesgo quirúrgico de acuerdo a la clasificación ASA.

RESULTADOS: De acuerdo con la clasificación ASA del riesgo quirúrgico, se agrupó a los pacientes en los siguientes grupos: 7 pacientes $(21,9 \%)$ presentaban ASA III y 25 pacientes $(78,1 \%)$ ASA IV. En 31 de 32 pacientes la cirugía se completó en una sesión. Interrogados acerca del dolor percibido, 11 pacientes refirieron no haber sentido dolor, 12 pacientes refirieron una molestia leve, y 3 pacientes un dolor esporádico. En los últimos tres pacientes se incrementó la dosis de sedación intravenosa con $0,1 \mathrm{mg} / \mathrm{kg}$ de peso corporal de Etomidato. El volumen medio de los fragmentos resecados fue de $47 \mathrm{ml}$, con un rango ente 18 y $120 \mathrm{ml}$, excepto en un paciente con un volumen de $205 \mathrm{ml}$ que precisó dos sesiones quirúrgicas.

CONCLUSIONES: La resección de próstatas de gran volumen en pacientes con riesgo quirúrgico se puede realizar con un mínimo de confort para el paciente utilizando una combinación de anestesia local y tubo de Amplatz suprapúbico. Este procedimiento es una opción a tener en cuenta como tratamiento alternativo a otros métodos.

PALABRAS CLAVE: Resección transuretral. Tubo de Amplatz. Anestesia local.

\section{ABSTRACT \\ "MODIFIED TECHNIQUE FOR TRANSURETHRAL PROSTATIC RESECTION USING A SUPRAPUBIC TUBE AND LOCAL ANAESTHESIA"}

PURPOSE: To establish a technique for transurethral resection of the prostate (TURP), combining the use of local anaesthesia with an Amplatz suprapubic tube, in patients at high surgical risk.

MATERIALS AND METHOD: A study was carried out in 32 patients who underwent TURP with a $30 \mathrm{~F}$ Amplatz suprapubic tube following local anaesthesia. This technique was indicated where surgery presented a general risk, in ederly patients, and for patients refusing to undergo spinal intradural or general anaesthesia. The mean age of the patients was 70 years (61-82 years). The risk of surgery was assessed according to the ASA classification.

RESULTS: According to the ASA classification, 7 patients were ASA III (21,9\%), and 25 patients were ASA IV (78,1\%). In 31 of the 32 patients the operation was completed in one session. When questioned about the pain they felt, 11 patients reported no pain, 12 slight discomfort and 3 ocassional pain. In the latter three patients, intravenous sedation was enhanced with $0,1 \mathrm{mg}$ etomidate per $\mathrm{kg}$ body weight. The volume of the resected fragments ranged from 18 to $120 \mathrm{ml}$, with a mean of $47 \mathrm{ml}$, except in one patient with $205 \mathrm{ml}$ who required two treatment sessions.

CONCLUSIONS: Large prostate resection in patients at high surgical risk was performed comfortably using a combination of local anaesthesia and an Amplatz suprapubic tube. This procedure is one possible option to be considered as an alternative to other treatments.

KEY WORDS: Transurethral resection. Amplatz tube. Local anaesthesia. 
$\mathrm{L}$ a resección transuretral de próstata es la técnica de elección en los pacientes con hiperplasia benigna de próstata que requieren tratamiento quirúrgico.

El uso del trocar de Reuter suprapúbico durante la intervención ayuda a reducir la morbilidad y proporciona un sistema de mantenimiento de baja presión de irrigación ${ }^{1}$. Así se consigue un mejor campo de visión y el tiempo operatorio puede extenderse permitiendo abordar próstatas de mayor volumen mediante técnicas endoscópicas.

Esto también permite la salida de los fragmentos por el drenaje conforme éstos son resecados.

En este estudio exponemos un procedimiento para desarrollar esta técnica bajo anestesia local y describimos los resultados obtenidos. Nuestro objetivo es desarrollar una cirugía segura y efectiva en pacientes con alto riesgo quirúrgico y pacientes con hipertrofias prostáticas importantes. Evaluamos la tolerancia de los pacientes, seguridad del método utilizado, y las posibles indicaciones.

\section{MATERIALES Y MÉTODOS}

Desde 1985, hemos llevado a cabo un total de 61 RTU de próstata bajo anestesia local, administrando el anestésico según distintos protocolos hasta que se halló el procedimiento más adecuado. A partir de 1992, utilizamos un tubo de Amplatz suprapúbico de gran calibre (30 Fr) en la mayoría de resecciones practicadas en nuestro centro. En este estudio presentamos los resultados de una serie de 32 pacientes intervenidos con anestesia local y colocación de un tubo de Amplatz suprapúbico.

La indicación de la RTU de próstata con anestesia local se basó en el riesgo quirúrgico general, que fue evaluado en base a la clasificación ASA.

La edad media de los pacientes fue de 70 años (rango de 61 a 82 años). Cuatro pacientes presentaban adenocarcinoma de próstata obstructivo. Cuatro pacientes presentaban litiasis vesical que se resolvió durante la misma intervención quirúrgica mediante litotricia electrohidráulica a través del tubo de Amplatz. Los pacientes fueron monitorizados y se estableció una vía venosa. Antes de la intervención se administraron 0,5 mg de Atropina y $5 \mathrm{mg}$ de Midazolam por vía intravenosa. Posteriormente no se administraron sedantes adicionales a menos que los pacientes experimentaran molestias.
Se infiltraron $40 \mathrm{ml}$ de Lidocaina en gel intrauretralmente, reteniendo la uretra con una pinza peneana. Con una aguja de Franzen, similar a la utilizada para la aspiración citológica de la próstata, se realizó una infiltración por vía transrectal de $5 \mathrm{ml}$ de Bupivacaína 0,25\% conteniendo un vasoconstrictor en la periferia de cada lóbulo prostático. A continuación infiltramos el área suprapúbica con $10 \mathrm{ml}$ de Mepivacaína al 2\% hasta la pared vesical.

Se utilizó un resector convencional de $28 \mathrm{~F}$ de calibre para llegar a la vejiga y realizar la uretrocistoscopia, para posteriormente conseguir la distensión vesical para colocar el tubo suprapúbico. Se inicia la intervención practicando una incisión cutánea suprapúbica de $1 \mathrm{~cm}$ a través de la cual se introduce un trocar de Reuter. A continuación se pasa un catéter ureteral de $7 \mathrm{~F}$ a través del trocar. Una vez retirado el trocar, se desliza un tubo de Amplatz con su correspondiente fiador sobre el catéter ureteral hasta situarlo dentro de la vejiga (Fig. 1). Todas las maniobras las realizamos con control endoscópico. Una vez retirados el catéter y el fiador, se conecta un tubo de $90 \mathrm{~cm}$ de polivinilo. Se considera que $90 \mathrm{~cm}$ es una distancia adecuada para producir la aspiración mediante la presión de la columna del líquido sin dañar la mucosa vesical. A medida que se resecan, los fragmentos pasan a través de este tubo hasta un filtro en forma de cesta (Fig. 2). Después del vaciado de la vejiga, cada lóbulo prostático se punciona con una aguja de Orandi que pasa a través del canal del elemento de trabajo del resector. Se infiltran $5 \mathrm{ml}$ de Bupivacaína al 0,25\% con vasoconstrictor en cada lóbulo.

La resección se practica mediante la técnica convencional, ayudada por la ausencia de sangrado y el alto flujo producido por el drenaje suprapúbico de $1 \mathrm{~cm}$ de diámetro. Dado que los fragmentos resecados son aspirados espontáneamente a través del tubo de Amplatz, la irrigación y aspiración de los fragmentos a través del resector no es necesaria, evitando así una recurrencia de la hemorragia.

Una vez finalizada la intervención, se pasa un tubo de Amplatz de $24 \mathrm{~F}$ cortado longitudinalmente a través del tubo suprapúbico, con objeto de introducir una sonda de Foley de $14 \mathrm{~F}$ a su través. Una sutura de seda cierra el ojal cutáneo y fija el tubo suprapúbico con una suave tracción 


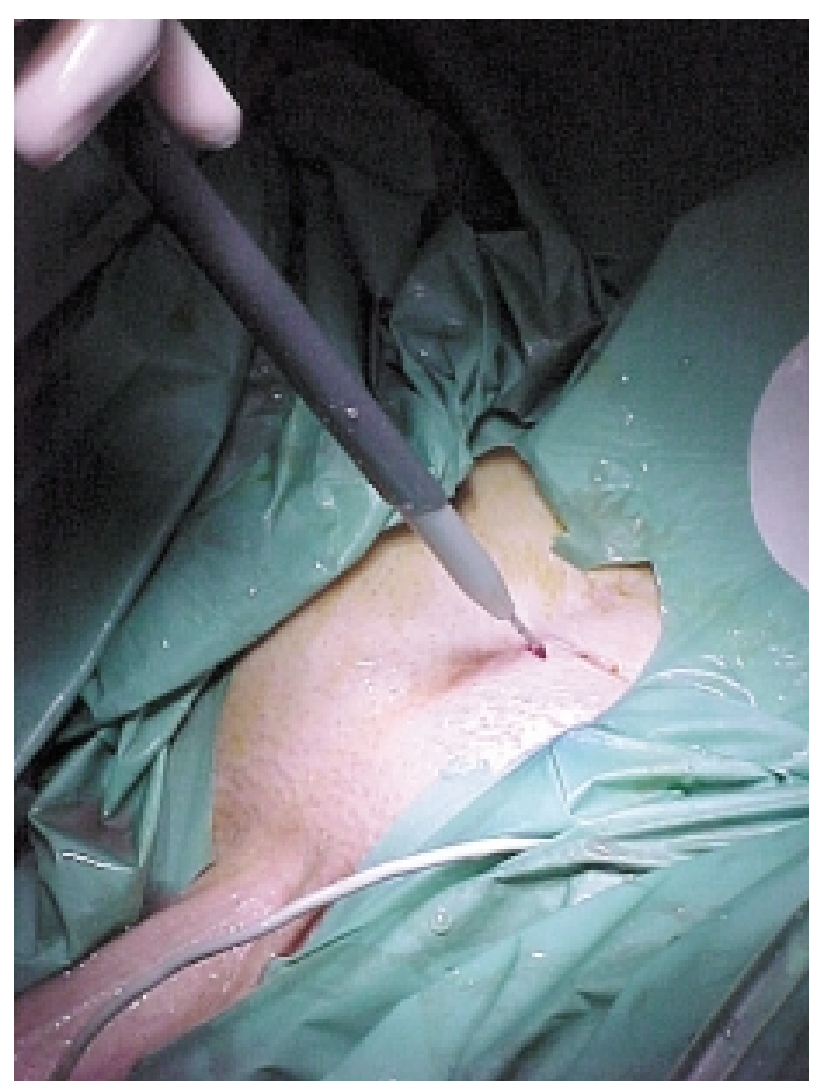

FIGURA 1. Introducción del Amplatz suprapúbico sobre el catéter ureteral.

para mantener el globo hinchado en la parte anterior de la vejiga. Por último se coloca una sonda uretral de 20-22 F.

Se determina la cantidad de tejido resecado en un matraz aforado mediante el método del volumen de líquido desplazado.

Tras la intervención, cada paciente fue interrogado para valorar el grado de tolerabilidad. Se pidió a los pacientes que evaluasen el dolor o la molestia percibidos en una escala del 0 al $10(0=$ ausencia total de dolor, y $10=$ dolor insoportable). También se les pidió que indicasen la satisfacción global con el procedimiento quirúrgico.

El tubo suprapúbico se retira 24 horas después de la intervención, y la sonda uretral a los cuatro días.

\section{RESULTADOS}

De acuerdo con la clasificación ASA del riesgo quirúrgico, se agrupó a los pacientes en los siguientes grupos: 7 pacientes $(21,9 \%)$ presentaban ASA III, y 25 pacientes (78,1\%) ASA IV.

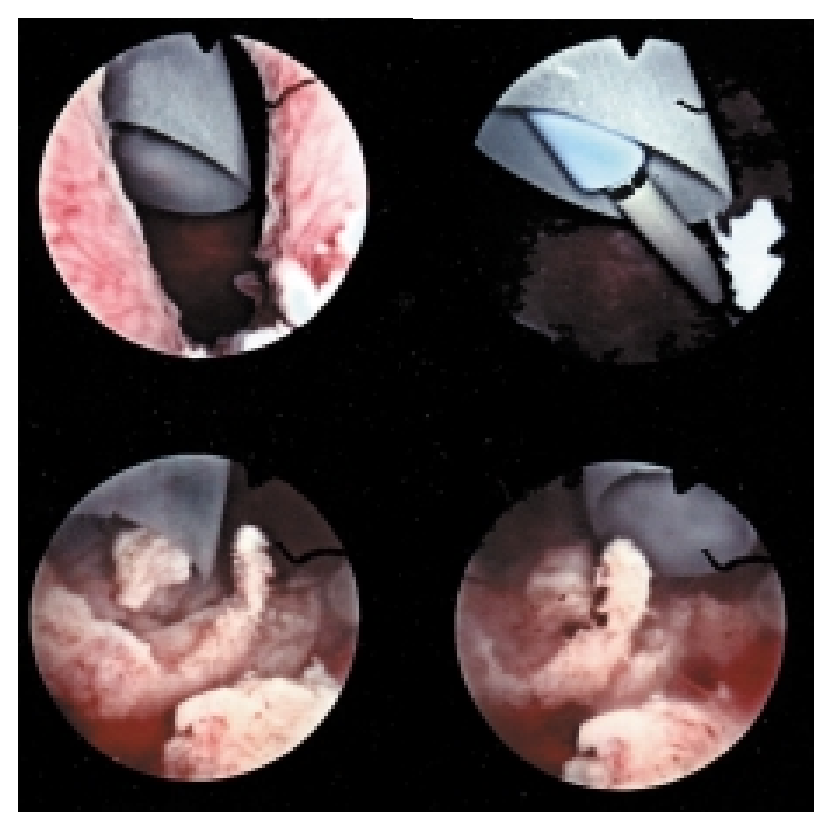

FIGURA 2. Fragmentos de resección saliendo espontáneamente a través del Amplatz.

En 31 de los 32 pacientes la intervención se llevó a cabo satisfactoriamente en una sola sesión. Ninguno de los 32 pacientes precisaron anestesia general. Sin embargo, la sedación intravenosa tuvo que potenciarse en 3 pacientes durante la intervención. Un paciente con un volumen prostático de $205 \mathrm{ml}$ necesitó dos sesiones quirúrgicas. El volumen de los fragmentos resecados, excluyendo el paciente anterior, varió entre $18 \mathrm{y}$ $120 \mathrm{ml}$, con una media de $47 \mathrm{ml}$. La administración de anestesia local y colocación del tubo suprapúbico requirió un tiempo medio de 12 minutos. La duración de la resección varió de 10 a 120 minutos, con una media de 42 minutos.

Ninguno de los pacientes necesitó transfusión sanguínea durante el periodo intraoperatorio o post-operatorio. No se observaron complicaciones importantes: no se produjeron fallecimientos, embolismos pulmonares o sepsis. No se produjeron perforaciones intestinales secundarias a la punción suprapúbica o fugas detectables del líquido de irrigación. Tras la retirada del tubo suprapúbico, la fístula cerró dentro de las primeras 24 horas en todos los pacientes.

En el cuestionario sobre el dolor, 11 pacientes indicaron una puntuación de 0 (ausencia de dolor), 13 indicaron una puntuación de 1 (molestia leve), y 3 una puntuación de 2 (dolor leve y 
esporádico). En los últimos tres pacientes, la sedación intravenosa se potenció con $0,1 \mathrm{mg}$ de Etomidato por Kg de peso corporal. En el cuestionario sobre el grado de satisfacción global, 26 pacientes declararon sentirse muy satisfechos, 4 estaban bastante satisfechos, y 2 no expresaron ninguna opinión. A la pregunta de si hubieran preferido haber sido intervenidos con otro tipo de anestesia, ninguno de los pacientes respondió afirmativamente.

\section{DISCUSIÓN}

La resección transuretral de próstata con anestesia local ha sido descrita por varios autores demostrando su aplicabilidad ${ }^{1,2}$. El uso de trocar de Reuter, práctica habitual en muchos centros, hace la resección más sencilla y aumenta la seguridad intraoperatoria ${ }^{1}$. Combinando ambas técnicas, hemos introducido los cambios que proponemos en este estudio. Utilizando una combinación de anestesia local asociada a vasoconstrictor y un drenaje suprapúbico de gran calibre, llevamos a cabo con rapidez y seguridad resecciones de adenomas, incluso de gran tamaño, en pacientes de alto riesgo quirúrgico.

En base al trabajo de Moffat y Sihna ${ }^{2,3}$, hemos utilizando durante más de 10 años anestesia local con grados variables de sedación para realizar la RTU de próstata en pacientes con alto riesgo quirúrgico ${ }^{4}$. Hemos intentado simplificar y mejorar el procedimiento anestésico buscando una combinación de varios fármacos que, debido a sus diferencias en las vías de metabolismo y eliminación, no alcanzarían en ningún momento niveles sanguíneos potencialmente tóxicos.

La vía de administración del anestésico puede ser transuretral, periuretral o intraprostática. Esta última se administra por vía transuretral, transperineal o transrectal. Moffat y Sinha utilizaron gel de lidocaína para anestesiar la mucosa uretral. Moffat también utilizó lidocaína asociada a epinefrina inyectada por vía percutánea en la uretra y luego infiltrada por vía perineal en la próstata $^{2}$, mientras que Sinha añadió una infiltración transuretral de los lóbulos prostáticos con bupivacaina ${ }^{3}$.

Hemos observado que el gel de lidocaína intrauretral perdió su eficacia durante la intervención. Esto no se produjo en el caso de la lido- caína inyectada, ni siquiera en los pacientes a los que no se practicaron infiltraciones periuretrales. Ninguno de los pacientes de este estudio recibió infiltraciones periuretrales.

Dado que en nuestro centro no se han registrado casos de prostatitis secundaria a punción con aguja fina, decidimos utilizar la aguja de Franzen para infiltrar selectivamente los pedículos prostáticos laterales cercanos a la cápsula. Este procedimiento es más seguro y preciso que la punción transperineal. El uso combinado de vasoconstrictor deja un área prostática sin irrigación, facilitando la resección y disminuyendo la hemorragia. Resulta destacable el hecho de que no fuera necesario realizar transfusiones sanguíneas en la resección de próstatas de más de $100 \mathrm{ml}$ de volumen de tejido resecado.

En 1992 Escovar comunicó el uso del tubo de Amplatz para extraer los fragmentos resecados de la vejiga. Comenzamos a utilizar dicho tubo en próstatas muy hipertróficas, pero al comprobar su eficacia sin aumentar la morbilidad su uso ha pasado a formar parte del procedimiento de rutina de la mayoría de resecciones realizadas en nuestro centro. El tiempo de colocación es inferior a un minuto y permite un flujo de irrigación que garantiza una visión óptima del campo durante la operación. El calibre del tubo permite mantener una presión de irrigación menor inferior a la presión venosa, previniendo el síndrome post-resección transuretral. Los fragmentos resecados salen espontáneamente; esto permite ahorrar tiempo y evita las hemorragias que se producen habitualmente durante la manipulación de la irrigación transuretral. La aspiración se produce por la columna del líquido en el tubo de drenaje, siendo proporcional a su longitud. Con una longitud de $90 \mathrm{~cm}$, el grado de aspiración obtenido extrae fácilmente incluso fragmentos litiásicos de varios milímetros sin dañar a la mucosa vesical. En este estudio, todas las fístulas suprapúbicas se cerraron. Además no hemos registrado casos de esta complicación en pacientes sometidos a RTU de próstata utilizando el tubo de Amplatz en nuestro centro.

Las ventajas de la utilización de anestesia local siempre que sea posible, evitando anestesia gene- 
ral o intradural son evidentes ${ }^{5,6}$. La anestesia general implica un riesgo para los pacientes con enfermedades cardiopulmonares. La anestesia espinal conlleva una sobrecarga de líquido intravascular que en ocasiones resulta difícil de manejar, especialmente cuando existe vasoparesia de los miembros inferiores durante las primeras horas después de la intervención.

La resección realizada de acuerdo con lo descrito es un procedimiento sencillo. Esta técnica permitió la resección completa del adenoma, dejando una uretra prostática amplia en los cuatro pacientes con adenocarcinoma prostático. Solamente un paciente necesitó dos sesiones debido al gran tamaño de la próstata.

Los pacientes con alto riesgo quirúrgico y que presentan síntomas prostáticos graves tributarios de cirugía, no deberían ser sometidos a una intervención que no garantizase una mejoría considerable en la mayor parte de los casos. Hasta este momento ninguna de las técnicas quirúrgicas ha demostrado ser más efectiva que la RTU de próstata ${ }^{7}$. Las modificaciones que proponemos en este estudio permiten su uso con un mínimo riesgo incluso en pacientes con grandes adenomas.

\section{REFERENCIAS}

1. REUTER HJ, JONES LW.: Physiologic low presure irrigation for transurethral resection: suprapubic trocar drainage. J Urol 1974; 111: 210-212.

2. MOFFAT NA.: Transurethral prostatic resections under local anaesthesia. J Urol 1977; 118: 607-608.

3. SINHA B, HAIKEL U, LANGE PH, MOON TD, NARAYAN P.: Transurethral resection of the prostate with local anaesthesia in 100 patients. $J$ Urol 1986; 135: 719-721.

4. VALDIVIA URÍA JG, LANCHARES SANTAMARÍA E, BLASCO BELTRÁN B, AMBROJ NAVARRO C, RAMÍREZ FABIÁN M, RODRÍGUEZ BAZALO JM.: ¿Hasta que punto puede disminuirse la morbilidad de la resección transuretral de próstata?. En: Jornadas de Urología Pierre Fabre, Madrid, Editores Médicos S.A. 1996: 17-31.

5. NIELSEN KK, ANDERSEN K, ASBJORN J, VORK F, OHRT-NISSEN A.: Blood loss in transurethral prostatectomy. Epidural versus general anaesthesia. Int Urol Nephrol 1987; 19 (3): 287-292.

6. MCGOWAN SW, SMITH GF.: Anaesthesia for transurethral prostatectomy: a comparison of spinal intradural analgesia with two methods of general anaesthesia. Anaesthesia 1980; 35 (9): 847-853.

7. VICENTE RODRÍGUEZ J.: Tratamiento de la HBP: realidades presentes y perspectivas futuras. Actas Urol Esp 2002; 26 (7): 481-490.

Dr. J.M. Sánchez Zalabardo

C/ San Francisco de Borja, 7 - 1ํㅡ

50009 Zaragoza

(Trabajo recibido el 7 octubre de 2002) 